\title{
CHANGE OF SIZE DISTRIBUTION OF POLYMER DROPLETS DURING THE SLURRY POLYMERIZATION OF STYRENE WITHIN ISOOCTANE DROPLETS SUSPENDED IN WATER
}

\author{
YASUO HATATE, HIROSHI HAMADA, ATSUSHI IKARI \\ AND FUMIYUKI NAKASHIO \\ Department of Chemical Engineering, Kagoshima University, Kagoshima 890
}

\begin{abstract}
Key Words: Styrene Slurry Polymerization, Microcapsule, Particle Size Distribution, Polymer Droplet, Chemical Reactor
\end{abstract}

\section{Introduction}

Styrene slurry polymerization within isooctane droplets suspended in water would be a useful microcapsule preparation method by in situ polymerization. ${ }^{1)}$ At the present time, this procedure shows no reproducible result at complete monomer conversion. This is caused by extremely low polymerization rates above $90 \%$ monomer conversion and the fluid property of the polymer shell of microcapsules. However, such a defect would be overcome by a charging initiator and/or hardening agent in the course of the polymerization. So, it is very important to know the behavior of polymer droplets during this polymerization.

In this study, the effect of important operating variables such as mixing rate and volume fraction of dispersed organic phase on the change of size distribution of polymer droplets with conversion were investigated to clarify the quantitative relationship between operating conditions and polymer droplets.

\section{Experimental}

\subsection{Reagent}

Reagents used in this work were prepared by the same method as mentioned previously. ${ }^{1,2)}$

\subsection{Experimental apparatus and procedure}

The same experimental apparatus as described in the previous paper ${ }^{1)}$ was employed, where the reactor was a $1000-\mathrm{ml}$ separable flask with four baffle blades having a width of $1 / 10$ the reactor diameter.

The experimental procedure is described below. Prescribed amounts of water and isooctane were charged into the reactor and the temperature in the reactor was raised to $70^{\circ} \mathrm{C}$ at a given rotation rate.

Received June 25, 1986. Correspondence concerning this article should be addressed to $Y$. Hatate. H. Hamada is now at Kao Co., Ltd., Wakayama 641. F. Nakashio is at Dept. of Org. Synth., Kyushu Univ., Fukuoka 812.
The initiator $\alpha, \alpha^{\prime}$-azobisisobutylonitrile (AIBN) was dissolved in a prescribed amount of styrene and was rapidly introduced into the reactor. From this time samples were taken at regular intervals. Microphotographs of the samples were taken to obtain the size distribution of polymer droplets. That is, the sample dropped in $5 \mathrm{wt} \%$ PVA aqueous solution on a glass plate was photographed with a microscope of 150-fold magnification. Each droplet in the photo was directly measured with a ruler. About 500 droplets were inspected for each sample. The Sauter average diameter of droplets was determined from the following equation.

$$
\bar{d}_{p_{32}}=\frac{\sum n_{i} d_{p_{i}}^{3}}{\sum n_{i} d_{p_{i}}^{2}}
$$

Size distribution of polymer droplets based on weight was calculated.

\section{Results and Discussion}

Experimental conditions are shown in Table 1.

To investigate the effect of rotation rate on the Sauter average diameter, the relations of $\bar{d}_{p_{32}}$ vs. conversion under the range of rotation rates from 450 to $1400 \mathrm{rpm}$ are shown in Fig. 1. $\bar{d}_{p_{32}}$ values of $30-60 \mu \mathrm{m}$ were obtained in the initial part of the reaction, which is reasonable in the range from 1400 to $450 \mathrm{rpm}$ according to the following equation of Vermeulen et $a l^{5}{ }^{5}$

$$
\frac{\bar{d}_{p_{32}}}{D}=0.055(1+3.3 \psi) W e^{-0.6}
$$

It is evident that the average diameter decreases clearly with increasing revolution rate and the average diameter increases with conversion except for the experiment at $1400 \mathrm{rpm}$. This peculiar relationship at $1400 \mathrm{rpm}$ may be due to the increasing production of extremely minute droplets with polymerization. 
Table 1. Experimental conditions

\begin{tabular}{cccccccccc}
\hline Run No. & \multicolumn{1}{c}{$\begin{array}{c}A_{0} \\
{[\mathrm{~mol} / l]}\end{array}$} & $\begin{array}{c}I_{0} \\
{[\mathrm{~mol} / l]}\end{array}$ & $\begin{array}{c}\psi_{0} \\
{[-]}\end{array}$ & $\begin{array}{c}\text { Rev. rate } \\
{\left[\mathrm{min}^{-1}\right]}\end{array}$ & $\begin{array}{c}\text { PVA } \\
{[\mathrm{wt} \%]}\end{array}$ & $\begin{array}{c}\text { Styrene } \\
{[\mathrm{g}]}\end{array}$ & $\begin{array}{c}\text { Isooctane } \\
{[\mathrm{g}]}\end{array}$ & $\begin{array}{c}\text { AIBN } \\
{[\mathrm{g}]}\end{array}$ & $\begin{array}{c}\text { Water } \\
{[\mathrm{g}]}\end{array}$ \\
\hline 3 & 5.15 & 0.051 & 0.16 & 650 & 0.1 & 77.2 & 35.9 & 1.21 & 739 \\
9 & 5.13 & 0.051 & 0.038 & 650 & 0.1 & 18.3 & 8.58 & 0.286 & 941 \\
4 & 3.01 & 0.060 & 0.14 & 650 & 0.1 & 39.5 & 52.8 & 1.24 & 841 \\
58 & 3.00 & 0.060 & 0.14 & 450 & 0.1 & 39.4 & 52.9 & 1.24 & 841 \\
59 & 3.00 & 0.060 & 0.14 & 1000 & 0.1 & 39.4 & 52.9 & 1.24 & 841 \\
60 & 3.00 & 0.060 & 0.14 & 1400 & 0.1 & 39.4 & 52.9 & 1.24 & 841 \\
\hline
\end{tabular}

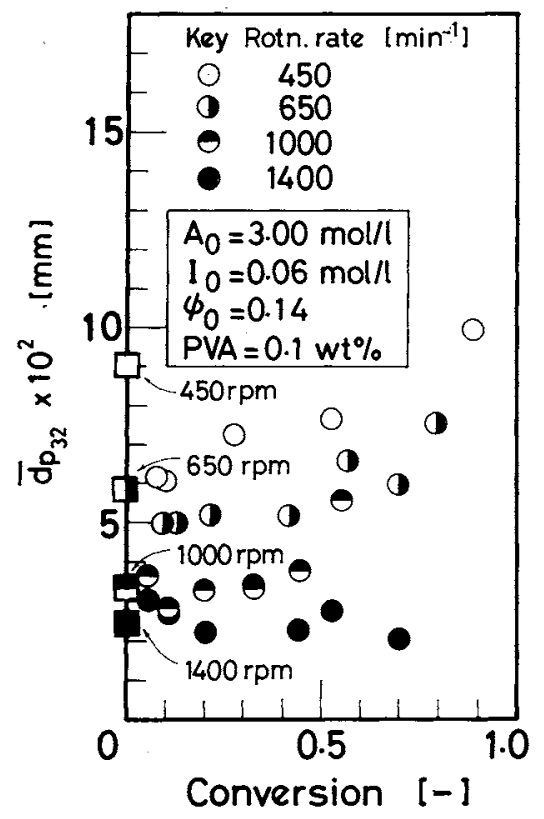

Fig. 1. Effect of rotation rate on average diameter. (Square keys are calculated by Eq. (2) using initial conditions. Each rotation rate used for calculation is shown for each key.)

The effects of rotation rate on size distribution of droplets at $A_{0}=3.0 \mathrm{~mol} / l$ are shown in Fig. 2. Here, IS, MS and FS represent the initial reaction stage (conversion $0-10 \%$ ), medial reaction stage (conversion $50-60 \%$ ) and final reaction stage (conversion $80-90 \%$, respectively. As predicted from the abovementioned result of the average diameter, the size distributions of droplets shift to the left with increasing rotation rate. At $1400 \mathrm{rpm}$, extremely minute droplets are found to occupy a much larger part at the medial stage of reaction than at the initial stage. The droplet size distribution in the initial reaction stage exhibits the sharp normal distribution. However, the normal distribution could not be predominant in the later stage because of the complicated coalescence and redispersion behavior of viscous droplets with increasing conversion.

The relations of $\bar{d}_{p_{32}}$ vs. conversion in the range of dispersion phase volume fraction from 0.04 to 0.16 is shown in Fig. 3 to demonstrate the effect of dispersion

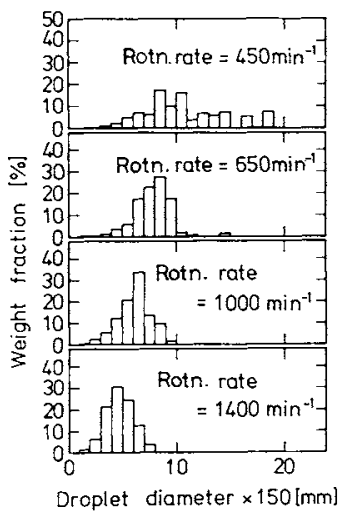

IS(conversion 0-10\%)

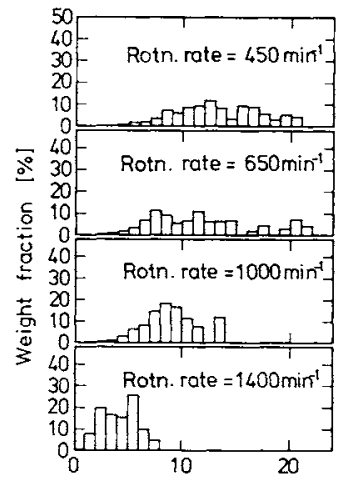

Droplet diometer $\times 150[\mathrm{~mm}]$

MS(conversion $50-60 \%$ )
Fig. 2. Effect of rotation rate on droplet size distribution

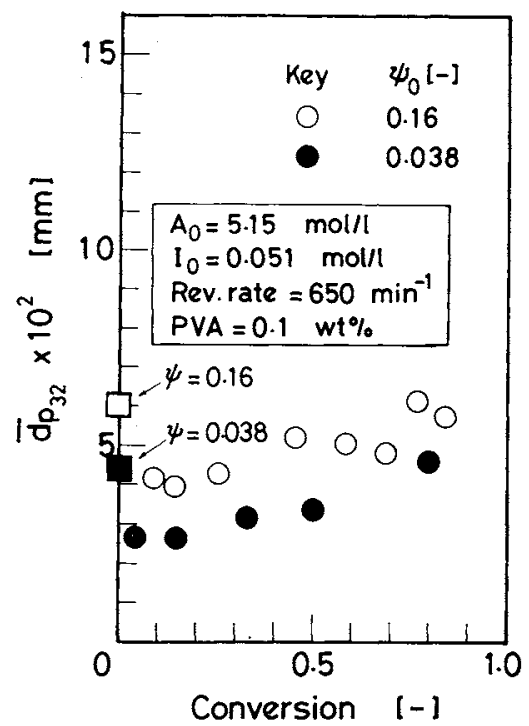

Fig. 3. Effect of dispersion phase volume fraction on average diameter. (Square keys are calculated by Eq. (2) using initial conditions. Each dispersion phase volume fraction is shown for each key.)

phase volume fraction on the average diameter. It is evident that the average diameter increases with increasing dispersion phase volume fraction and monomer conversion.

The effect of dispersion phase volume fraction on droplet size distributions at $A_{0}=5.1 \mathrm{~mol} / l$ is shown in 

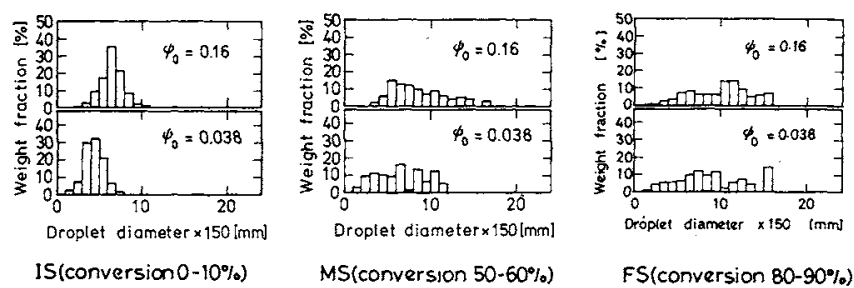

Fig. 4. Effect of dispersion phase volume fraction on droplet size distribution.

Fig. 4. The size distribution shifts to the right with increasing dispersion phase volume fraction, and becomes broader as the polymerization proceeds.

\section{Conclusions}

To clarify the behavior of polymer droplets in styrene slurry polymerization within suspended isooctane droplets in water, the Sauter average diameter and size distribution of polymer droplets were studied. The following results were obtained.

1) Although a polymer shell would be formed around each droplet in the course of the polymerization, ${ }^{1)}$ almost the same effects of rotation rate and dispersion phase volume fraction as in suspension polymerization ${ }^{3,4)}$ were observed for the change of droplet size with conversion. The average diameter greatly decreases with rotation rate and slightly increases with dispersion phase volume fraction.

2) The normal size distribution is predominant for droplets in the initial reaction stage. However, in the later stage of reaction, such a normal size distribution is not maintained any longer due to complicated behavior of droplets with different redisper- sion and coalescence rates and polymerization rates.

$$
\begin{aligned}
& \text { Nomenclature } \\
& \text { A } \quad=\text { monomer concentration in dispersed phase } \\
& \text { [mol/l] } \\
& D \quad=0.05 \text {, impeller diameter } \quad[\mathrm{m}] \\
& d_{p_{32}} \quad=\text { Sauter average diameter } \quad[\mu \mathrm{m}] \\
& I=\text { initiator concentration in dispersed phase } \\
& \text { PVA = polyvinyl alcohol concentration in }[\mathrm{mol} / \mathrm{l}] \\
& \text { We water phase [mol/l] } \\
& \psi \quad=\text { dispersion phase volume fraction }
\end{aligned}
$$

\section{Literature Cited}

1) Hatate, Y., Y. Kurokawa, H. Hamada, A. Ikari and F. Nakashio: J. Chem. Eng. Japan, 18, 293 (1985).

2) Hatate, Y., T. Hano, T. Miyata, F. Nakashio and W. Sakai: Kagaku Kōgaku, 35, 903 (1971).

3) Hirose, M. and E. Ohshima: Kagaku Kōgaku, 34, 181 (1970).

4) Takao, M., S. Matsumoto and T. Imoto: Kobunshi Kagaku, 29, 811 (1972).

5) Vermeulen, T., G. M. Williams and G. E. Langlois: Chem. Eng. Progr., 51, 85-F (1955). 